\title{
Boundary Layer Analysis of the Ridge Singularity in a Thin Plate.
}

\author{
Alexander E. Lobkovsky \\ The James Franck Institute \\ The University of Chicago \\ 5640 South Ellis Avenue Chicago, Illinois 60637
}

(October 15, 2018)

\begin{abstract}
Large deformations of thin elastic plates and shells present a formidable problem in continuum mechanics which is generally intractable except by numerical methods. Conventional approaches break down in the limit of small plate thickness due to appearance of discontinuities in the solution which require boundary layer treatment. We examine a simple case of a plate bent by forces exerted along its boundary so as to create a sharp crease in the limit of infinitely small thickness. We find a separable boundary layer solution of the von Karman plate equations which is valid along the ridge line. We confirm a scaling argument [- [h that the ridge possesses a characteristic radius of curvature $R$ given by the thickness of the sheet $h$ and the length of the ridge $X$ viz. $R \sim h^{1 / 3} X^{2 / 3}$. The elastic energy of the ridge scales as $E \sim \kappa(X / h)^{1 / 3}$ where $\kappa$ is the bending modulus of the sheet. We determine the dependence of these quantities on the dihedral angle of the ridge $\pi-2 \alpha$. For all angles $R \sim \alpha^{-4 / 3}$ and $E \sim \alpha^{7 / 3}$. The framework developed in this paper is suitable for determination of other properties of ridges such as their interaction or behavior under various types of loading. We expect these results to have broad importance in describing forced crumpling of thin sheets.
\end{abstract}

\section{INTRODUCTION}

Mechanical properties of a thin elastic sheet undergoing large distortions is a rich problem which is still largely unresolved despite great effort [2]. The postbuckling behavior of thin-walled structures is of importance in safety design and in development of energy absorbing structures [3,4]. Some microscopic solid-like membranes can also be found in a crumpled state. Examples of such membranes include phospholipid bilayers below the $2 \mathrm{D}$ freezing point [5], networks of tropomyosin [6], and monomolecular layers such as graphite oxide [7] or molybdenum disulfide [8]. In an entirely different context, formation of mountain ranges is a result of strong deformation of earth's tectonic plates which to an extent behave as elastic sheets [9].

Several types of distortion in elastic membranes have been recently analyzed. Thermal fluctuations of a flat membrane roughen the surface, but preserve its overall flat shape [10]. The surface is thought to lose its flatness for sufficiently high temperature, at the "crumpling transition." By contrast, certain defects deliberately introduced into flat membrane destroy its flatness. The energy associated with these distortions is well understood [11]. Different distortions occur when a flat membrane is collapsed by an external force. The incipient deformation in response to a load and the associated buckling instabilities are classic subjects of continuum mechanics [12,13.

Large deformations of thin plates and shells can be described by two coupled quadratic fourth order partial differential equations whose explicit solution exists only in a handful of cases [14]. The main complication is, however, that the small parameter related to the thickness of the shell usually multiplies the highest derivative or the nonlinear term in the equations [15]. This leads to formation of boundary layers in the asymptotic limit of small shell thickness. An example in which such boundary layer appears is a thin plate bent by torques applied at the boundary first analyzed by Kelvin, Tait and Friedrichs [20]. Other well-known boundary layer phenomena which include the Prandtl's boundary layer in a flow at large Reynolds numbers [16] arise under similar conditions.

The novel character of the boundary layer problem considered in this article is in its potential relevance to a broad range of crumpling phenomena. A number of empirical studies have investigated the statistical scaling properties of the crumpled state 17 19. Current theoretical understanding of this forced crumpling is in its initial stages, however. It was argued in Ref. 21] that the structure of a thin crumpled sheet can be thought of as a collection of flat facets bound by a network of straight ridges which terminate at sharp vertices. These ridges have the same nature as the boundary layer arising in a simple geometry considered in this article. The scaling properties of the boundary layer solution allow one to make far-reaching conclusions about the morphology and the elastic energy of a crumpled thin sheet.

In the next section we derive the equations which describe large deflections of thin elastic plates and introduce the boundary value problem which exhibits the novel boundary layer. Scaling properties of this boundary layer are examined in Section III. A separation of variables Ansatz is introduced in Section IV. The Ansatz implies that the local properties of the ridge solution scale with the distance from the boundary in a simple way. In Section $\mathrm{V}$ we derive the scaling of the separable solution with the dihedral angle of the ridge $\alpha$. From here on we will refer to $\alpha$ as the "dihedral angle" even though it is a half of the difference of the dihedral angle from $\pi$. Confusion is unlikely to result since this is the only way $\alpha$ 
is used in the paper. Corroborating numerical evidence is presented in Section VI. Finally, the implications of the thickness scaling for the crumpling problem and the validity of the separable solution as well as future work are discussed in Section VII.

\section{VON KARMAN PLATE EQUATIONS}

We start with a brief review of the theory of large deflections of thin plates originally due to von Karman [22]. The equations are usually obtained within an expansion scheme of the full three dimensional elasticity equations with the thickness of the plate as a small parameter. Small strains and a linear stress-strain relation are usually assumed [23. We however prefer to derive the equations in a somewhat different way which is more instructive while not as rigorous as the thickness expansion method.

When the thickness of the plate is small the in-plane stresses are much greater than the normal stresses, so that the dependence of the in-plane stresses on the normal coordinate is simple 12. Then, all variables can be integrated over the thickness of the plate and it can be treated as a two-dimensional surface. The plate has a preferred material coordinate system $\mathbf{x} \in D$ some open simply connected domain in $\mathbf{R}^{2}$. According to the Fundamental Theorem of Surfaces [24], to define a surface uniquely up to an overall translation and rotation in $\mathbf{R}^{3}$ it is sufficient to specify two symmetric tensors in $D$ : a metric tensor $g_{\alpha \beta}$ and a curvature tensor $C_{\alpha \beta}$ satisfying a set of relations which we will write down shortly. To clarify the meaning of these tensors we first note that the strain tensor $\gamma_{\alpha \beta}$ is defined as the deviation of the metric tensor from the identity

$$
g_{\alpha \beta}=\delta_{\alpha \beta}+2 \gamma_{\alpha \beta}
$$

The sum of the eigenvalues of the strain tensor $\gamma_{1}+\gamma_{2}$ is the (2D) expansion (or compression) factor and their difference is the shear angle [12]. The eigenvalues of the curvature tensor, on the other hand, are the inverses of the two principal radii of curvature of the surface. Given the three-dimensional position $\overrightarrow{\mathbf{r}}\left(x_{1}, x_{2}\right)$ of the material point $\left(x_{1}, x_{2}\right)$ the metric tensor and the curvature tensor are given by 24]

$$
\begin{aligned}
g_{\alpha \beta} & =\left(\partial_{\alpha} \overrightarrow{\mathbf{r}}\right) \cdot\left(\partial_{\beta} \overrightarrow{\mathbf{r}}\right) \\
C_{\alpha \beta} & =\hat{\mathbf{n}} \cdot\left(\partial_{\alpha} \partial_{\beta} \overrightarrow{\mathbf{r}}\right),
\end{aligned}
$$

where $\partial_{\alpha}$ denotes differentiation with respect to the material coordinate $x_{\alpha}$, and $\hat{\mathbf{n}}$ is the unit normal to the surface. To be absolutely rigorous one must distinguish upper and lower indices but since we define all quantities to first nontrivial order in the strain $\gamma_{\alpha \beta}$ and raising and lowering of the indices is accomplished by applying the metric tensor $g_{\alpha \beta}$, raising and lowering indices only affects higher order terms in $\gamma_{\alpha \beta}$.
In order for the tensors $g_{\alpha \beta}$ and $C_{\alpha \beta}$ to define a surface they must satisfy two relations involving the Christoffel symbols $\Gamma_{\alpha \beta \mu}$ [24] which are defined in terms of the metric tensor. When the strains are small the expression is particularly simple:

$$
\Gamma_{\alpha \beta \mu}=-\partial_{\mu} \gamma_{\alpha \beta}+\partial_{\alpha} \gamma_{\beta \mu}+\partial_{\beta} \gamma_{\alpha \mu}
$$

The first relation which $g_{\alpha \beta}$ and $C_{\alpha \beta}$ must satisfy in order to define a surface is the Gauss's Theorema Egregium [24]. It expresses the Gaussian curvature $K=\operatorname{det} C_{\alpha \beta}$ which is the determinant of the curvature tensor in terms of the Christoffel symbols. In the case of small and slowly varying strain (i.e. $\left.\partial^{2} \gamma \gg(\partial \gamma)^{2}\right)$ the Gauss's theorem reads

$$
\begin{aligned}
K & =\partial_{2} \Gamma_{211}-\partial_{2} \Gamma_{212}+\Gamma_{11 \rho} \Gamma_{\rho 22}-\Gamma_{12 \rho} \Gamma_{\rho 21} \\
& =\partial_{\alpha} \partial_{\beta} \gamma_{\alpha \beta}-\nabla^{2} \operatorname{tr} \gamma_{\alpha \beta},
\end{aligned}
$$

where $\nabla^{2}=\partial_{\mu} \partial_{\mu}$. Summation over repeated indices is implied. Geometrically, the Gauss's equation captures the intuitive notion that non-zero Gaussian curvature (the sheet curves in both directions) must cause the sheet to strain. Due to historical reasons Eq. (5) in this context is usually referred to as the first von Karman equation.

The other set of relations usually termed as CodazziMainardi equations describes how the curvature tensor behaves when transported around a closed curve. Again, when the strains and consequently the Christoffel symbols are small these relations are simple. They say that if the curvature tensor is parallel transported around a closed curve, the change is of higher order in the strain 24

$$
\partial_{\gamma} C_{\alpha \beta}=\partial_{\beta} C_{\alpha \gamma}
$$

These equations are a tensor analogy of the condition satisfied by an irrotational vector field. And just as an irrotational vector field can be written as a gradient of a scalar, there exists a scalar function $f$ such that

$$
C_{\alpha \beta}=\partial_{\alpha} \partial_{\beta} f
$$

Equations Eq. (5) and (6) ensure that $C_{\alpha \beta}$ and $\gamma_{\alpha \beta}$ describe a physical surface. We now consider further conditions which assure that each element of the surface is in mechanical equilibrium. The forces and the torques acting on an infinitesimal element of the surface $\delta x \delta y$ can be found from the the stress tensor $\sigma_{\alpha \beta}$ and the torques $M_{\alpha \beta}$ 114 as shown in Fig. 1a and 1b. The force tensors are related to the deformation tensors $\gamma_{\alpha \beta}$ and $C_{\alpha \beta}$ by the constitutive relations for an elastic body. For sufficiently small strains,

$$
\sigma_{\alpha \beta}=\frac{Y}{1-\nu^{2}}\left[\gamma_{\alpha \beta}+\nu \epsilon_{\alpha \rho} \epsilon_{\beta \tau} \gamma_{\rho \tau}\right]
$$

where $Y$ is the Young's modulus and $\nu$ is the Poisson ratio of the elastic material [12]. Here $\epsilon_{\alpha \beta}$ is the two 
dimensional antisymmetric tensor. There is a similar relation for the torques per unit length

$$
M_{\alpha \beta}=\kappa\left[C_{\alpha \beta}+\nu \epsilon_{\alpha \rho} \epsilon_{\beta \tau} C_{\rho \tau}\right]
$$

which comes from considering the stress distribution throughout the thickness of the plate $h$ [14]. Here $\kappa=Y h^{3} /\left(12\left(1-\nu^{2}\right)\right)$ is the bending rigidity of the plate 12. The in-plane force equilibrium can be written down by inspection (see Fig. 1a). It reads

$$
\partial_{\alpha} \sigma_{\alpha \beta}=0 \text {. }
$$

This condition allows one to write the stresses in terms of a scalar potential $\chi$ traditionally called the force function:

$$
\sigma_{\alpha \beta}=\epsilon_{\alpha \mu} \epsilon_{\beta \nu} \partial_{\mu} \partial_{\nu} \chi
$$

This relation is a tensor analog of the divergenceless vector field expressed as a curl of a vector potential.

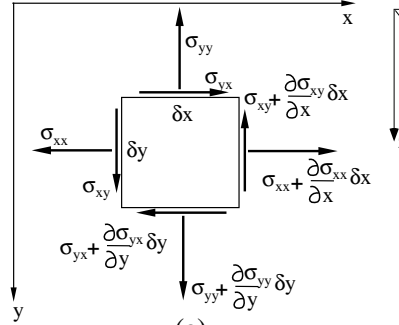

(a)

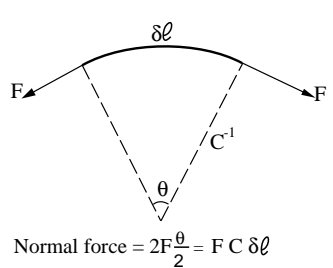

(c)

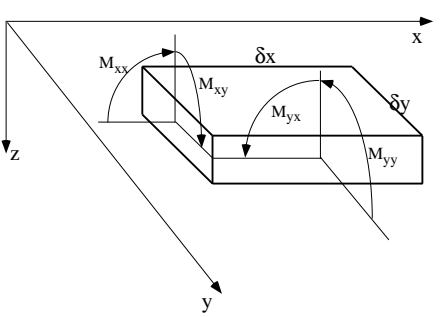

(b)

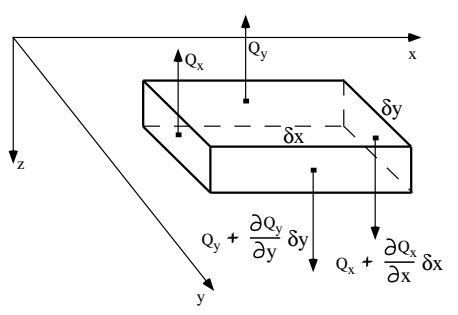

(d)
FIG. 1. (a) In-plane stresses (per unit length) acting on a small element of an elastic sheet, (b) Torques per unit length acting on the same element, (c) One-dimensional derivation of the normal force on a curved element $\delta l$ due to in-plane forces $F$, (d) Normal shear stresses acting over the sides of the elementary area of the sheet.

The resultant normal force on the element of the surface can come from three sources. First, is the external distributed load which we for the time being will ignore. Second, the normal components of the in-plane tensions that act on the element are non-zero due to the curvature of the surface. It is easy to become convinced (see Fig. 1c for a one dimensional version of the derivation) that that normal force per unit area due to the in-plane tension is $h \sigma_{\alpha \beta} C_{\alpha \beta}$ [14]. Third, changing torques in the sheet produce a normal force. To see this, we define the normal shear stresses $Q_{\alpha}$ acting over the sides of our surface element as shown on Fig. 1d. The normal force on the element due to the variation of these shear stresses is clearly $\partial_{\alpha} Q_{\alpha} \delta x \delta y$. The normal shear stresses $Q_{\alpha}$ can be found by balancing the moments about the $x$ - and $y$ axes of all the forces acting on the element which gives $Q_{\alpha}=\partial_{\beta} M_{\alpha \beta}$. Collecting the normal forces we obtain the second von Karman equation

$$
\partial_{\alpha} \partial_{\beta} M_{\alpha \beta}=h \sigma_{\alpha \beta} C_{\alpha \beta} .
$$

The more familiar form of the von Karman equations emerges when one substitutes the potentials $f$ and $\chi$ into Eqs. (5) and (12) using Eq. (8), Eq. (9), and the definition of the potentials. They read

$$
\left\{\begin{aligned}
\kappa \nabla^{4} f & =[\chi, f] \\
\frac{1}{Y} \nabla^{4} \chi & =-\frac{1}{2}[f, f],
\end{aligned}\right.
$$

where we have defined

$$
\begin{aligned}
{[a, b] } & \equiv \epsilon_{\alpha \mu} \epsilon_{\beta \nu}\left(\partial_{\alpha} \partial_{\beta} a\right)\left(\partial_{\mu} \partial_{\nu} b\right) \\
& =\frac{\partial^{2} a}{\partial x^{2}} \frac{\partial^{2} b}{\partial y^{2}}+\frac{\partial^{2} a}{\partial y^{2}} \frac{\partial^{2} b}{\partial x^{2}}-2 \frac{\partial^{2} a}{\partial x \partial y} \frac{\partial^{2} b}{\partial x \partial y} .
\end{aligned}
$$

Notice that $[f, f]$ is twice the Gaussian curvature $K$.

In principle, the strains $\gamma_{\alpha \beta}$ and the curvatures $C_{\alpha \beta}$ obtained from the von Karman equations as a function of the material coordinates define the surface uniquely (up to position in $\mathbf{R}^{3}$ ). The problem of finding the shape of the surface from the strains and the curvatures is however highly nonlinear and intractable in general. On can make progress in a limited class of deformations in which the normal to the surface does not change much. In that case the so called Monge coordinates are appropriate. The undeformed plate is located in the $x-y$ plane so that upon deformation the point originally at $\left(x_{1}, x_{2}, 0\right)$ moves to $\left(x_{1}+u_{1}, x_{2}+u_{2}, w\right)$ in the 3 -dimensional embedding space where $u_{\alpha}$ and $w$ are functions of $x_{\alpha}$. If the derivatives of $u_{\alpha}$ and $w$ are small everywhere, only the lowest non-trivial order terms in those derivatives can be kept in the expressions for the strains and the curvatures. We obtain 12

$$
\gamma_{\alpha \beta}=\frac{1}{2}\left(\partial_{\alpha} u_{\beta}+\partial_{\beta} u_{\alpha}+\partial_{\alpha} w \partial_{\beta} w\right)
$$

and

$$
C_{\alpha \beta}=\partial_{\alpha} \partial_{\beta} w
$$

It is clear that the normal displacement $w$ in this case is precisely the potential function $f$ defined in Eq. (7).

To complete the description of the deformation of the thin elastic plate one needs to be able to calculate the elastic energy stored in the sheet and specify the boundary conditions on the functions $f$ and $\chi$. We begin by considering the work done on the small element of the surface $\delta x \delta y$ by the surrounding parts of the plate when the strain in the element changes by $\delta \gamma_{\alpha \beta}$. That work 
is $h \sigma_{\alpha \beta} \delta \gamma_{\alpha \beta} \delta x \delta y$ [14]. The stretching energy $E_{s}$ in the plate element is found by integrating the strain from 0 to its value $\gamma_{\alpha \beta}$ while keeping in mind that the stresses are proportional to the strains which introduces a factor of $1 / 2$. Thus the total stretching energy in the plate is given by

$$
E_{s}=\frac{h}{2} \int d x d y \sigma_{\alpha \beta} \gamma_{\alpha \beta}
$$

One can similarly show that the work done by the torques $M_{\alpha \beta}$ in bending a surface element is $\frac{1}{2} M_{\alpha \beta} C_{\alpha \beta} \delta x \delta y$ so that the total bending energy $E_{b}$ in the plate is 14

$$
E_{b}=\frac{1}{2} \int d x d y M_{\alpha \beta} C_{\alpha \beta}
$$

Expressing the strains, stresses, torques and curvatures in terms of the potentials $\chi$ and $f$ we get after some algebra

$$
\begin{aligned}
& E_{s}=\frac{h}{2 Y} \int d x d y\left[\operatorname{tr}\left(\partial_{\alpha} \partial_{\beta} \chi\right)\right]^{2}-2(1+\nu) \operatorname{det}\left(\partial_{\alpha} \partial_{\beta} \chi\right) \\
& E_{b}=\frac{\kappa}{2} \int d x d y\left[\operatorname{tr}\left(\partial_{\alpha} \partial_{\beta} f\right)\right]^{2}-2(1-\nu) \operatorname{det}\left(\partial_{\alpha} \partial_{\beta} f\right)
\end{aligned}
$$

One can show that the conformation $\mathbf{r}\left(x_{1}, x_{2}\right)$ of the sheet which is a solution of the von Karman equations minimizes the elastic energy while satisfying the boundary conditions 23. We note in passing that many derivations of the von Karman equations (see e. g. Ref. [11]) start with writing down these energies in an add-hoc way and then taking a variational derivative with respect to the sheet shape.

We are left to address the question of the boundary conditions. As pointed out in Ref. [23], specification of the boundary conditions for the von Karman equations is tricky and is rarely done in a completely rigorous or even correct manner. The main problem arises when a fixed shape of the boundary in the embedding space is prescribed. Since the equations are in terms of the material coordinates, the knowledge of the shape in the embedding space requires solving the equations first. Therefore, a boundary condition that makes reference to the embedding space is intractable. Only in the case when the material coordinates can be replaced with the Monge coordinates mentioned above can one prescribe a fixed shape the boundary. In other cases the best one can do is to specify the curvature tensor and the strain tensor at the boundary of the material coordinates domain. One can imagine pulling or pushing on the edge of the plate which amounts to specifying the stresses at the edges. Torques $M_{\alpha \beta}$ and normal forces $Q_{\beta}$ can also be applied at the edge.

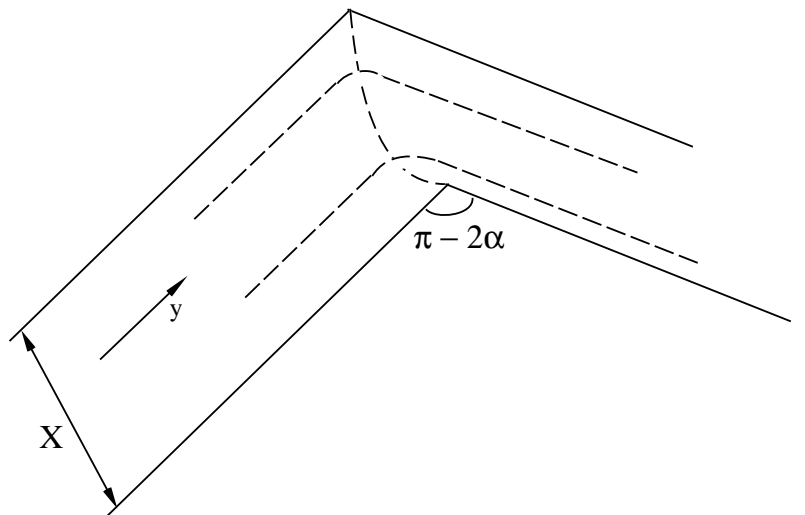

FIG. 2. Long strip of width $X$ bent through a dihedral angle $\alpha$.

We proceed to define the boundary value problem which exhibits the ridge singularity. We consider a strip $(x, y) \in(-X / 2, X / 2) \times(-\infty, \infty)$ of uniform thickness $h$ made of isotropic homogeneous material with Young's modulus $Y$ and Poisson ratio $\nu$. Normal forces are applied to the edge so as to bend the strip by an angle $\pi-2 \alpha$ (see Fig. 2). The membrane stresses $\sigma_{\alpha \beta}$ as well as the torques $M_{\alpha \beta}$ vanish at the boundary (except for the singular point $y=0$ ). In terms of the potentials $f$ and $\chi$ it means that

$$
\partial_{\alpha} \partial_{\beta} f=\partial_{\alpha} \partial_{\beta} \chi=0 \quad \text { at } \quad x= \pm \frac{X}{2} .
$$

The condition that the strip is bent translates into specifying the curvature tensor at the boundary. We assume that the boundary is a geodesic of the surface which is reasonable in the small strain limit. In the direction along the boundary the curvature is zero except for a sharp peak at the origin $y=0$ where the strip is bent. The width of the bent region must be of the order of the thickness of the plate and the curvature is of the order of the inverse thickness. It is convenient then to set the curvature along the boundary to a $\delta$-function since the all the length scales in the problem are much larger than $h$. This leads to a particularly simple condition on $f$ at the boundary

$$
f( \pm X / 2, y)=\alpha|y|
$$

up to an arbitrary linear function of $x_{\alpha}$. To see that the coefficient $\alpha$ is identical to the bending angle introduced above consider the following integral along the boundary (or any other geodesic which approaches $x=$ const)

$$
\frac{\partial f}{\partial y}(\infty)=\int_{0}^{\infty} d y \frac{\partial^{2} f}{\partial y^{2}}=\int_{0}^{\infty} d y\left|\frac{\partial \hat{\mathbf{n}}}{\partial y}\right|=\alpha,
$$

where we have used the definition of the curvature tensor $C_{y y}=\partial_{y} \hat{\mathbf{n}} \cdot \hat{\mathbf{t}}_{y}$ and the fact that the integral is taken along a geodesic. Here $\hat{\mathbf{n}}$ is the unit normal and $\hat{\mathbf{t}}_{y}$ is tangent vector to the surface in the $y$-direction [24]. The geometric meaning of this integral is the length of the contour 
which is inscribed by the end of the normal vector $\hat{\mathbf{n}}$ on the unit sphere as $\hat{\mathbf{n}}$ is transported along the geodesic. Using this argument and a reasonable belief that the solution far away from the ridge approaches that of an unperturbed flat strip on can deduce that the derivative of the potential $f$ along the transverse direction $y$ does not depend on $x$ far away from the ridge. This fact will later allow us to gain insight into the nature of the solution.

The physical reason one expects non-trivial singular behavior in the small thickness limit is the interplay between the bending and the stretching energies. The key observation is that the bending modulus $\kappa$ vanishes faster with the thickness of the plate (as $h^{3}$ ) than the stretching modulus $Y h$ [12] so that thin plates bend in preference to stretching. Therefore, in the limit of zero thickness, the bent plate develops a sharp crease at which the solution is irregular (the curvature in the $y$-direction diverges). At a finite thickness, as shown in ref. [1], the crease softens to a characteristic width governed by the competition of the bending and the stretching energies in the region of the crease.

\section{BOUNDARY LAYER SCALING}

We first put the von Karman equations (13) in the non-dimensional form by defining

$$
\bar{\chi}=h \frac{\chi}{\kappa}, \quad \bar{f}=\frac{f}{X}, \quad \bar{x}=\frac{x}{X} \quad \text { and } \quad \bar{y}=\frac{y}{X}
$$

to obtain

$$
\left\{\begin{aligned}
\nabla^{4} \bar{f} & =[\bar{\chi}, \bar{f}] \\
\lambda^{2} \nabla^{4} \bar{\chi} & =-\frac{1}{2}[\bar{f}, \bar{f}]
\end{aligned}\right.
$$

with the small dimensionless geometry parameter

$$
\lambda \equiv \frac{\sqrt{\kappa / Y}}{X}=\left(\frac{h}{X}\right) \frac{1}{\sqrt{12\left(1-\nu^{2}\right)}}
$$

multiplying the highest derivative. The solution to the so-called reduced problem defined by $\lambda=0$ can be readily obtained. There is no cost for bending and thus the strip froms a sharp crease $f(x, y)=\alpha|y|$. The properties of the boundary layer which corrects the discontinuity in the reduced solution be found by the rescaling

$$
\tilde{f}=\lambda^{\beta} \bar{f}, \quad \tilde{\chi}=\lambda^{\delta} \bar{\chi}, \quad \tilde{x}=\lambda^{0} \bar{x}, \quad \tilde{y}=\lambda^{\beta} \bar{y} .
$$

Note that $\bar{x}$ remains unchanged by the rescaling transformation and $\bar{f}$ and $\bar{y}$ are rescaled by the same factor to satisfy the boundary condition 22 . The rescaled equations read:

$$
\left\{\begin{array}{c}
\lambda^{-\beta}\left[\frac{\partial^{4} \tilde{f}}{\partial \tilde{x}^{4}}+2 \lambda^{2 \beta} \frac{\partial^{4} \tilde{f}}{\partial^{2} \tilde{x} \partial^{2} \tilde{y}}+\lambda^{4 \beta} \frac{\partial^{4} \tilde{f}}{\partial^{4} \tilde{y}}\right]=\lambda^{\beta-\delta}[\tilde{\chi}, \tilde{f}] \\
\lambda^{2-\delta}\left[\frac{\partial^{4} \tilde{\chi}}{\partial \tilde{x}^{4}}+2 \lambda^{2 \beta} \frac{\partial^{4} \tilde{\chi}}{\partial^{2} \tilde{x} \partial^{2} \tilde{y}}+\lambda^{4 \beta} \frac{\partial^{4} \tilde{\chi}}{\partial^{4} \tilde{y}}\right]=\lambda^{0} \frac{1}{2}[\tilde{f}, \tilde{f}] .
\end{array}\right.
$$

The dominant terms in the $\lambda \rightarrow 0$ limit must be of the same order on either side of the equations. This leads unambiguously to $\beta<0$ which is in agreement with the intuitive guess that the width of the boundary layer must go to zero as the thickness of the sheet vanishes. Balance of the dominant terms gives

$$
\beta=-\frac{1}{3} \quad \text { and } \quad \delta=\frac{2}{3}
$$

in agreement with the scaling argument of Witten and Li. With the knowledge of the scaling behavior of the functions $f$ and $\chi$ on can obtain the small thickness behavior of other quantities such as, for example, the transverse ridge curvature $C_{y y}=\partial^{2} f / \partial y^{2}$ and the midridge longitudinal strain $\gamma_{x x}=(1 / Y)\left(\sigma_{x x}-\nu \sigma_{y y}\right) \simeq(1 / Y) \partial^{2} \chi / \partial y^{2}$ where the expressions are evaluated at $x=0$ and $y=$ 0 . The leading order behavior of the transverse stress $\sigma_{y y}=\partial^{2} \chi / \partial x^{2}$ is of higher order in $\lambda$ and it thus can be ignored in the expression for $\gamma_{x x}$. Substituting the rescaled quantities we find that

$$
C_{y y}=\frac{1}{X} \lambda^{-1 / 3} \frac{\partial^{2} \tilde{f}}{\partial \tilde{y}^{2}} \quad \text { and } \quad \gamma_{x x}=\lambda^{2 / 3} \frac{\partial^{2} \tilde{\chi}}{\partial \tilde{y}^{2}}
$$

These expressions give the asymptotic behavior of $C_{y y}$ and $\gamma_{x x}$ since the rescaled quantities do not depend on $\lambda$ in the small $\lambda$ limit.

Note that the width of the boundary layer has the same leading order behavior as the radius of curvature of the plate at the center of the ridge and as the "sag" which is the vertical deflection of the ridge shape from that of perfectly sharp crease. This deflection is given by $f(0,0)$ in the small dihedral angle limit $\alpha \ll 1$. The sag can be found for a general dihedral angle $\alpha$ from the following argument. Due to the $x \rightarrow-x$ symmetry of the solution the ridge line $y=0$ is a geodesic of the surface. Therefore we can relate the vertical deflection of the sheet $w(x)$ along the ridge to the curvature $C_{x x}=\partial^{2} f / \partial x^{2}$. To lowest order in the strain we obtain

$$
\frac{w^{\prime \prime}}{\sqrt{1-\left(w^{\prime}\right)^{2}}}=C_{x x}=\frac{\partial^{2} f}{\partial x^{2}} .
$$

Since $C_{x x}$ vanishes in the limit of small thickness, the leading term in the $\lambda^{2 / 3}$ expansion of $w^{\prime \prime}(x)$ scales with the same power of $\lambda$ as $\partial^{2} f / \partial x^{2}$. Thus the ridge sag $w(0)$ is of the same order as the ridge width and as the midridge transverse radius of curvature.

We can also find asymptotic behavior of the bending and stretching energies of the sheet. We first note that in the expressions for the energies Eqs. (19) and (20) the 
terms involving $\operatorname{det}\left(\partial_{\alpha} \partial_{\beta} f\right)$ and $\operatorname{det}\left(\partial_{\alpha} \partial_{\beta} \chi\right)$ can be expressed as an integral over the boundary [12]. These integrals vanish identically for the boundary conditions considered here. Substituting the rescaled quantities which remain finite as $\lambda \rightarrow 0$ into Eqs. (19) and (20) we obtain

$$
\begin{aligned}
& E_{b}=\kappa \lambda^{-1 / 3} \int d \tilde{x} d \tilde{y}\left(\frac{\partial^{2} \tilde{f}}{\partial \tilde{y}^{2}}\right)^{2} \\
& E_{s}=\kappa \lambda^{-1 / 3} \int d \tilde{x} d \tilde{y}\left(\frac{\partial^{2} \tilde{\chi}}{\partial \tilde{y}^{2}}\right)^{2},
\end{aligned}
$$

in agreement with the energy scaling argument of Witten and $\mathrm{Li}$ [1]. For a fixed thickness, these energies grow qualitatively slower (as $X^{1 / 3}$ ) than the energy of a sharp crease of size $X$ which grows linearly with $X$.

\section{A SOLUTION TO THE REDUCED EQUATIONS}

The solution to the rescaled equations (19) can be sought as an expansion in powers of $\lambda^{2 / 3}$,

$$
\left\{\begin{array}{l}
\tilde{f}=f_{0}+\lambda^{2 / 3} f_{1}+\lambda^{4 / 3} f_{2}+\ldots \\
\tilde{\chi}=\chi_{0}+\lambda^{2 / 3} \chi_{1}+\lambda^{4 / 3} \chi_{2}+\ldots
\end{array}\right.
$$

Plugging the series into Eq. (28) and matching the coefficients of powers of $\lambda^{2 / 3}$ we can obtain equations for all orders in the expansion Eq. (34). We get at zeroth order,

$$
\left\{\begin{array}{l}
\frac{\partial^{4} f_{0}}{\partial \tilde{y}^{4}}=\left[\chi_{0}, f_{0}\right] \\
\frac{\partial^{4} \chi_{0}}{\partial \tilde{y}^{4}}=-\frac{1}{2}\left[f_{0}, f_{0}\right] .
\end{array}\right.
$$

We note in passing the equations for $f_{1}$ and $\chi_{1}$. They read

$$
\left\{\begin{array}{l}
\frac{\partial^{4} f_{1}}{\partial \tilde{y}^{4}}+2 \frac{\partial^{4} f_{0}}{\partial \tilde{x}^{2} \partial \tilde{y}^{2}}=\left[\chi_{0}, f_{1}\right]+\left[\chi_{1}, f_{0}\right] \\
\frac{\partial^{4} \chi_{1}}{\partial \tilde{y}^{4}}+2 \frac{\partial^{4} \chi_{0}}{\partial \tilde{x}^{2} \partial \tilde{y}^{2}}=-\left[f_{0}, f_{1}\right] .
\end{array}\right.
$$

At this point we must draw the readers' attention to the fact that while $\tilde{f}$ and $\tilde{\chi}$ satisfy the boundary conditions Eq. (21) or Eq. (22) there is no reason to expect that $f_{0}$ and $\chi_{0}$ do. In other words, it is likely that the expansion in powers of $\lambda^{2 / 3}$ does not converge uniformly, so that for a fixed $\lambda$ higher orders in the expansion Eq. (34) become increasingly important as the boundary is approached. A solution of the zeroth order equations (35) might therefore be a good approximation to $\tilde{f}$ and $\tilde{\chi}$ only in a restricted area around the ridge away from the boundaries.

To help solve equations (35) we observe that the boundary conditions do not introduce another length scale to the problem. Therefore, the transverse profile of the ridge ought to scale with the distance from its midpoint $\tilde{x}=0$. We must find a functional form of $f_{0}$ and $\chi_{0}$ such that the different $\tilde{x}=$ const slices are related to each other by a scale factor $q(\tilde{x})$ depending on $\tilde{x}$ only. By assuming scaling in $\tilde{x}$ we hope to decouple it from the new transverse variable $\xi=\tilde{y} / q(\tilde{x})$. We assume that

$$
f_{0}(\tilde{x}, \xi)=q^{\mu} p_{1}(\xi), \quad \chi_{0}(\tilde{x}, \xi)=q^{\eta} p_{2}(\xi) .
$$

As we show in the appendix, variables separate for only one choice of $\eta=\mu=1$ which means that the width of the ridge solution scales with the distance from the vertex in the same way as the ridge sag. As mentioned above, the boundary conditions on $f$ require that its transverse derivative $\partial f / \partial y$ approach a constant independent of $x$. If a similar condition is to hold for $f_{0}$ then inescapably $\mu=1$. The validity of this scaling hypothesis needs to be corroborated by some other means. In Section VI we present numerical evidence supporting this scaling Ansatz, here we only note that this evidence is convincing.

Substitution of the Ansatz Eq. (37) into the zeroth order equations (35) gives

$$
\left\{\begin{array}{l}
p_{1}^{\prime \prime \prime \prime}=q^{\prime \prime} q^{2}\left[p_{1}^{\prime \prime} p_{2}+p_{2}^{\prime \prime} p_{1}-\xi\left(p_{1}^{\prime \prime} p_{2}^{\prime}+p_{2}^{\prime \prime} p_{1}^{\prime}\right)\right] \\
p_{2}^{\prime \prime \prime \prime}=-q^{\prime \prime} q^{2}\left[p_{1}^{\prime \prime} p_{1}-\xi p_{1}^{\prime \prime} p_{1}^{\prime}\right]
\end{array}\right.
$$

Separation of variables provides the equation for the scale factor $q^{\prime \prime} q^{2}=A$, where $A$ is some constant. This equation, together with the condition that $q(x)$ be even can be solved by a substitution $g(q)=q^{\prime}(\tilde{x})$ to yield,

$$
\tilde{x}=\frac{1}{\pi}\left[\frac{\pi}{2}-\arcsin \sqrt{\frac{q(\tilde{x})}{q(0)}}+\sqrt{\frac{q(\tilde{x})}{q(0)}\left(1-\frac{q(\tilde{x})}{q(0)}\right)}\right]
$$

where the separation of variable constant is related to $q(0)$ via $A=-\pi^{2} q^{3}(0) / 2$. The scale factor $q(\tilde{x})$ has a singular derivative near the vertices, since for $\tilde{x}=1 / 2-\epsilon$

$$
q(\epsilon) \simeq\left(\frac{3 \pi}{2}\right)^{2 / 3} \epsilon^{2 / 3},
$$

which reinforces the suspicion that the series expansion (34) does not converge uniformly.

Therefore to test the predicted functional form of the scale factor one ought to look a local property of the numerical solution away from the boundary and find its limit as $\lambda \rightarrow 0$. We discuss such a solution in Section VI. Numerically one has access to only a limited range of thickness to size ratios. ( $\lambda$ cannot be too small otherwise lattice effects become significant.) Nevertheless, the Taylor expansion coefficients of the scale function $q(\tilde{x})$ around $\tilde{x}=0$ can be successfully extracted from the numerics. Since there is one adjustable parameter $q(0)$ we must look at the first two coefficients in the expansion $q(\tilde{x})=q(0)\left(1+b_{2} \tilde{x}^{2}+b_{4} \tilde{x}^{4}+\ldots\right)$. Expanding Eq. (39) we find $b_{2}=\pi^{2} / 4 \simeq 2.47$ and $b_{4}=\pi^{4} / 48 \simeq 2.03$, whereas the numerics gives $b_{2}=2.53 \pm 0.04$ and $b_{4}=2.09 \pm 0.07$ 
where the errors are obtained from the scatter of the data.

The transverse equations (38) can be integrated once by defining

$$
\phi_{\beta}(\xi)=p_{\beta}(\xi)-\xi p_{\beta}^{\prime}(\xi) \quad \text { for } \quad \beta=1,2
$$

We obtain

$$
\left\{\begin{array}{l}
\phi_{1}^{\prime \prime}-\frac{2}{\xi} \phi_{1}^{\prime}=A \phi_{1} \phi_{2}+D_{1} \\
\phi_{2}^{\prime \prime}-\frac{2}{\xi} \phi_{2}^{\prime}=-\frac{1}{2} A \phi_{1}^{2}+D_{2} .
\end{array}\right.
$$

Where $D_{\beta}$ are the integration constants allowing for a non zero asymptote of the functions $\phi_{\beta}$. The finite limit of these functions as $\xi \rightarrow \infty$ would imply a finite stress and curvature far away from the ridge. However, since we expect the series Eq. (34) to converge non-uniformly, the large $\xi$ behavior of $\phi_{\beta}$ is likely to be unphysical. The functions $p_{\alpha}$ can then be readily found from

$$
p_{\beta}(\xi)=G_{\beta} \xi+\xi \int_{\xi}^{\infty} d \zeta \frac{\phi_{\beta}(\zeta)}{\zeta^{2}},
$$

where $G_{\beta}$ are integration constants. A further analysis of the transverse equations will be done elsewhere. Here we only remark that the simplicity and the apparent symmetry of the Eqs. (42) must point to some physical symmetry in the problem which forces this behavior to occur. Perhaps further light can be shed onto the physical meaning of the functions $\phi_{\beta}$ by writing down the expression for the elastic energy of the sheet in terms of the separable solution. The leading term in the $\lambda^{2 / 3}$-expansion of the total energy as in Eq. (33) reads

$$
\begin{aligned}
E & =\int d \tilde{x} d \tilde{y} \frac{1}{q^{2}}\left(\left(p_{1}^{\prime \prime}\right)^{2}+\left(p_{2}^{\prime \prime}\right)^{2}\right) \\
& =\int \frac{d \tilde{x}}{q(\tilde{x})} \int \frac{d \xi}{\xi^{2}}\left[\left(\frac{d \phi_{1}}{d \xi}\right)^{2}+\left(\frac{d \phi_{2}}{d \xi}\right)^{2}\right]
\end{aligned}
$$

We can also make contact with the boundary condition $\tilde{f} \rightarrow \alpha|\tilde{y}|$ as $\tilde{y} \rightarrow \infty$. If the integral in Eq. (43) converges, this boundary condition gives $G_{1}=\alpha$. Now one can use the $y \rightarrow-y$ symmetry of the problem and sufficient smoothness of functions involved to conclude that $p_{1}^{\prime}(0)=0$ which leads to

$$
\int_{0}^{\infty} \frac{d \zeta}{\zeta} \frac{d \phi_{1}(\zeta)}{d \zeta}=-\alpha
$$

\section{DIHEDRAL ANGLE SCALING OF THE SEPARABLE SOLUTION}

Even without having solved the transverse equations one can deduce the behavior of the solution for different dihedral angles $\alpha$ just from the form of the Eqs. (42).
We begin by noting that there exists a two-parameter family of transformations which produce new solutions. For example if $\vec{\phi}(\xi)$ is a two-component solution, then $\vec{\psi}(\xi)=S_{1} \vec{\phi}\left(S_{2} \xi\right)$ is also a solution of the equations (42) but with a different separation of variable constant $A^{\prime}=S_{2}^{2} / S_{1} A$. We must allow for variation of $A$ since it will undoubtedly depend on the dihedral angle. Using the boundary condition on $\phi_{1}$ Eq. (45) we can find the corresponding dihedral angle $\alpha^{\prime}=S_{1} S_{2} \alpha$. With this condition, there is a one-parameter family of scale transformations which produce a solution for the dihedral angle $\alpha^{\prime}$ given that for the dihedral angle $\alpha$. Let us fix a reference solution $\vec{\phi}_{0}$ with $\alpha=1$ so that all other solutions are labeled by the scale factors $S_{1}$ and $S_{2}$. We can now find how various quantities of interest are affected by the scale transformation. We start with the simplest cases

$$
\begin{aligned}
\alpha & \sim S_{1} S_{2} \\
p_{1} \sim p_{2} & \sim S_{1} \\
q \sim A^{1 / 3} & \sim S_{1}^{-1 / 3} S_{2}^{2 / 3} .
\end{aligned}
$$

The relevant quantities such as the ridge sag $q p_{1}$, the transverse curvature in the middle of the ridge $(1 / q) p_{1}^{\prime \prime}$ both evaluated at $\tilde{x}=\xi=0$ and the elastic energy Eq. (44) all turn out to depend only on the product of the scaling factors $S_{1} S_{2}$ which determines unambiguously their scaling with the dihedral angle $\alpha$. Using Eqs. (45) we get

$$
\begin{aligned}
& q p_{1} \\
& \begin{aligned}
(1 / q) p_{1}^{\prime \prime} & \sim S_{1}^{1 / 3} S_{2}^{-2 / 3} S_{1} S_{2}^{2} \sim \alpha^{4 / 3} \\
\int \frac{d \tilde{x}}{q(\tilde{x})} \int \frac{d \xi}{\xi^{2}}\left(\frac{d \phi}{d \xi}\right)^{2} & \sim S_{1}^{1 / 3} S_{2}^{-2 / 3} S_{1}^{2} S_{2}^{3} \sim \alpha^{7 / 3} .
\end{aligned} \\
& \sim S_{1}^{-1 / 3} S_{2}^{2 / 3} S_{1} \sim \alpha^{2 / 3}
\end{aligned}
$$

One can also predict how the width of the ridge which scales with $S_{2}^{-1}$ depends on the dihedral angle $\alpha$ using one extra assumption. If the transverse curvature decays exponentially away from the ridge then the width of the ridge scales as the radius of curvature times the bend angle $\alpha$. Therefore

$$
\text { ridge width } \sim S_{2}^{-1} \sim \alpha^{-1 / 3} .
$$

This equation fixes the dependence of the scale factors $S_{1}$ and $S_{2}$ on $\alpha$ so that solutions for all dihedral angles can be generated from a single solution for some $\alpha$. All of the $\alpha$-scaling predictions have been convincingly verified by the numerics.

\section{NUMERICAL SIMULATION OF THE STRETCHING RIDGE}

We modeled an elastic sheet as a triangular lattice of springs of unstretched length $a$ and spring constant $K$ after Seung and Nelson [11]. Bending rigidity is introduced 
by assigning an energy $J\left(1-\hat{\mathbf{n}}_{1} \cdot \hat{\mathbf{n}}_{2}\right)$ to every pair of adjacent lattice triangles with normals $\hat{\mathbf{n}}_{1}$ and $\hat{\mathbf{n}}_{2}$. Seung and Nelson showed that when strains are small and radii of curvature are large compared to the lattice constant, this model membrane bends and stretches as a sheet of thickness $h=a \sqrt{8 J / K}$ made of isotropic homogeneous material with Young's modulus $Y=2 K a /(h \sqrt{3})$ and Poisson ratio $\nu=1 / 3$. The bending modulus is $\kappa=J \sqrt{3} / 2$. We use a conjugate gradient energy minimization routine to obtain a sequence of relaxed shapes with the varying geometric parameter $\lambda$. This lattice model has been used by the author and others to verify the $\lambda$-scaling [21]. Here we explore the other two types of scaling which we are convinced govern the ridge properties.

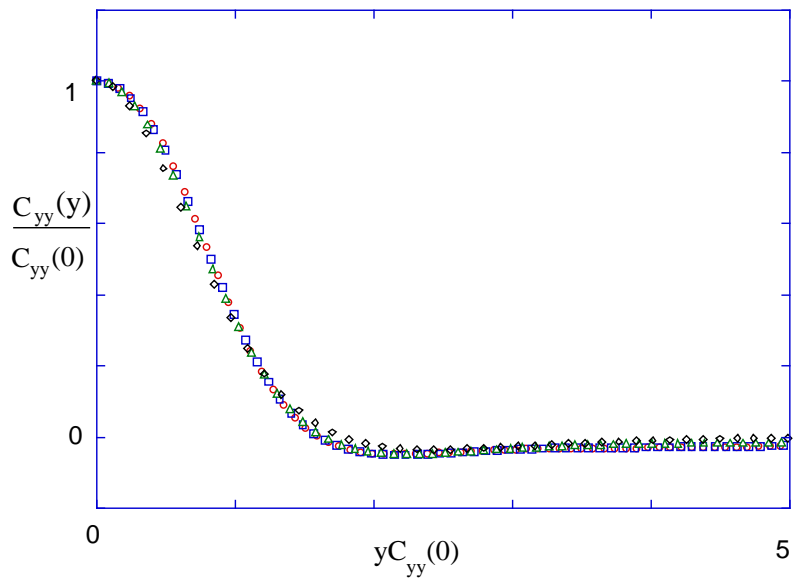

FIG. 3. Transverse curvatures $C_{y y}(x, y)$ for $x=0$ (circles), $x=5$ (squares), $x=10$ (triangles) and $x=15$ (diamonds) each scaled by $C_{y y}(x, 0)$ vs. the transverse coordinate $y$ scaled by $C_{y y}^{-1}(x, 0)$. The curvatures are found numerically from a $50 a$ by $500 a$ strip bent by a 90 degree angle.

A rather suggestive demonstration of the $q(\tilde{x})$-scaling is presented on Fig. 3. We plot the transverse curvatures for different slices $\tilde{x}=$ const obtained from a simulated strip of dimensions $50 a$ by $500 a$ bent by normal forces applied to the $x= \pm X / 2$ parts of the boundary to make a 90 degree angle. The dimensionless thickness is $\lambda \simeq 10^{-3}$. The curvature for each slice is scaled by its value at the origin $\tilde{y}=0$. The transverse coordinate is scaled by the inverse of the curvature at $\tilde{y}=0$. These profiles collapse onto a single scaling curve. A more quantitative test is based on the prediction for the variation of the transverse radius of curvature along the ridge (i. e. at $\xi=0) R_{y y}=1 / C_{y y} \sim q(\tilde{x})$. The coefficients $b_{2}$ and $b_{4}$ in the expansion $R_{y y}(\tilde{x}) \simeq R_{y y}(0)\left(1+b_{2} \tilde{x}^{2}+b_{4} \tilde{x}^{4}\right)$ can be extracted from the numerics and compared to those obtained from Eq. (39).

Here we can also test the dependence of the scaling on the boundary conditions which cause the ridge singularity. Instead of using a long strip bent by normal forces applied to the boundaries we can consider any shape which in the limit of zero thickness exhibits a sharp crease. One such shape chosen due to ease of implementation is a regular tetrahedron which was previously used to verify the $\lambda$-scaling [21]. A picture of one such minimum-energy tetrahedral surface is presented in Fig. 4. Shading is proportional to the local stretching energy.

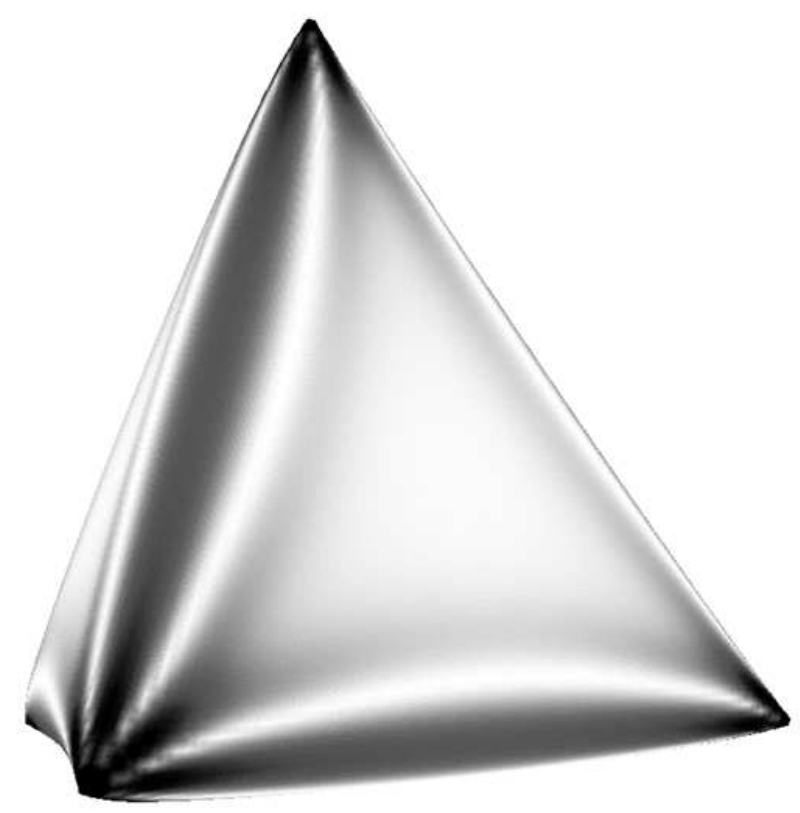

FIG. 4. A tetrahedron of side $X=100 a$ and thickness $h=0.063 a$. Shading is proportional to the local stretching energy. Note the "sagging" of the ridge.

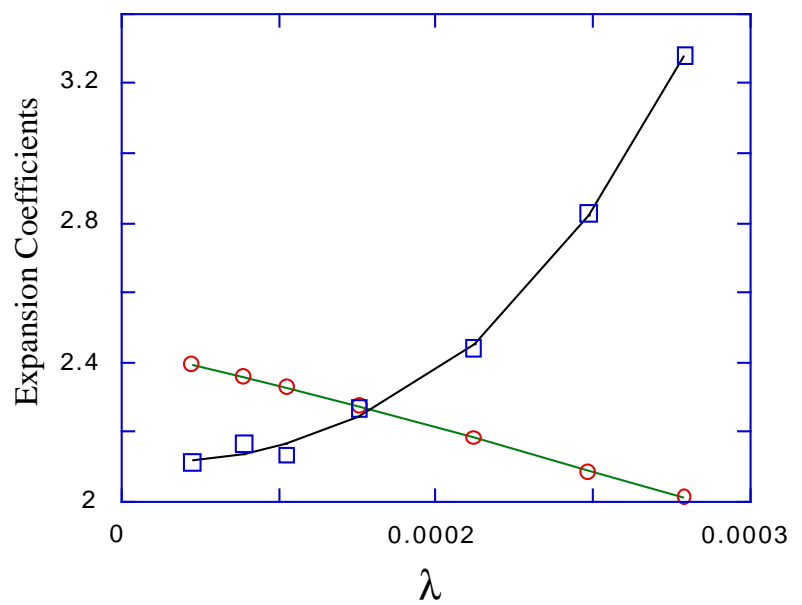

FIG. 5. Scale factor expansion coefficients $b_{2}$ (circles) and $b_{4}$ (squares) as extracted from a least squares fit to the functional form of the transverse radius of curvature along the ridge for tetrahedra of $X=100 a$.

Figure 5 displays a plot of the coefficients $b_{2}$ (circles) and $b_{4}$ (squares) versus the dimensionless thickness $\lambda$ obtained by a least squares fit to the functional forms of $R_{y y}$ in the range of $|\tilde{x}| \leq 0.2$ for tetrahedra of varying thicknesses but fixed edge length $X=100 a$. We see that 
these coefficients have a well defined limit as $\lambda \rightarrow 0$ which is approached algebraically. The extrapolations to $\lambda=0$ give $b_{2}=2.53 \pm 0.04$ and $b_{4}=2.09 \pm 0.07$ as compared to the prediced values of $b_{2} \simeq 2.47$ and $b_{4} \simeq 2.03$.

We tested the dihedral angle scaling by making a long rectangular strip of dimensions $50 a$ by $200 a$ and applying normal forces to its long boundaries so as to bend it by an angle $\alpha$. For all angles we fixed $\lambda \simeq 5 \times 10^{-4}$. The results are displayed in Figures 6 and 7. Fig. 6 is a plot of the total elastic energy in units of the bending modulus $\kappa$ vs. the anticipated scaling variable $\alpha^{7 / 3}$. To a good precision the energy does indeed exhibit the predicted scaling behavior. The deviation at the small bending angles is due to the finite size effects. Since the width of the ridge diverges as $\alpha \rightarrow 0$ larger sheets are needed for smaller angles to avoid finite size effects. In Fig. 7 the midridge curvature $C_{y y}(0)$ in units of $X^{-1}$ is plotted against $\alpha^{4 / 3}$. The data agree well with the scaling prediction.

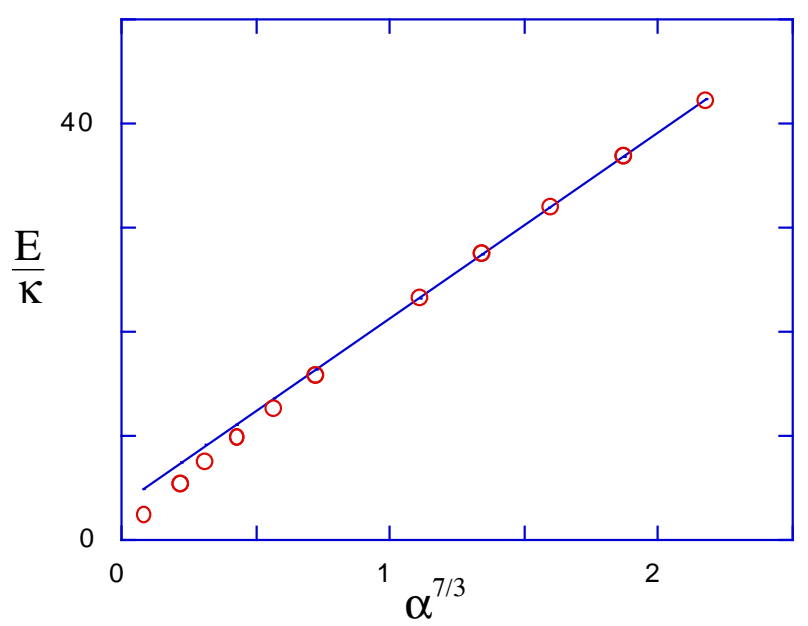

FIG. 6. Total elastic energy in units of the bending modulus $\kappa$ of $50 a$ by $500 a$ strips of thickness $h=0.063 a$ bent by an angle $\alpha$.

Another test relevant to the question of the boundary condition dependence of the scaling predictions is the comparison of the coefficients $\mathrm{R}$ for different boundary conditions in the asymptotic form of the total elastic energy of the ridge $E / \kappa \simeq R \lambda^{-1 / 3} \alpha^{7 / 3}$. In a preceding work [21] we found this coefficient for the ridge appearing in the tetrahedral shape described above. Its value, which was found by examining the dependence of the energy on $\lambda$ for a fixed dihedral angle, is $R_{t e t}=1.161 \pm 0.003$. For the long strip we have found $R_{\text {strip }}=1.24 \pm 0.01$ by fixing $\lambda$ and varying $\alpha$. Here the error range reflects only the uncertainty arising from scatter in the numerical data. However, there are additional errors resulting from corrections to the asymptotic scaling not properly accounted for in our crude fitting procedure. We therefore suspect that the scaling coefficient $R$ depends insensitively on the boundary conditions and varies only by a fraction of its value. A stronger claim of boundary condition independence of $R$ would require a more convincing demonstra- tion.

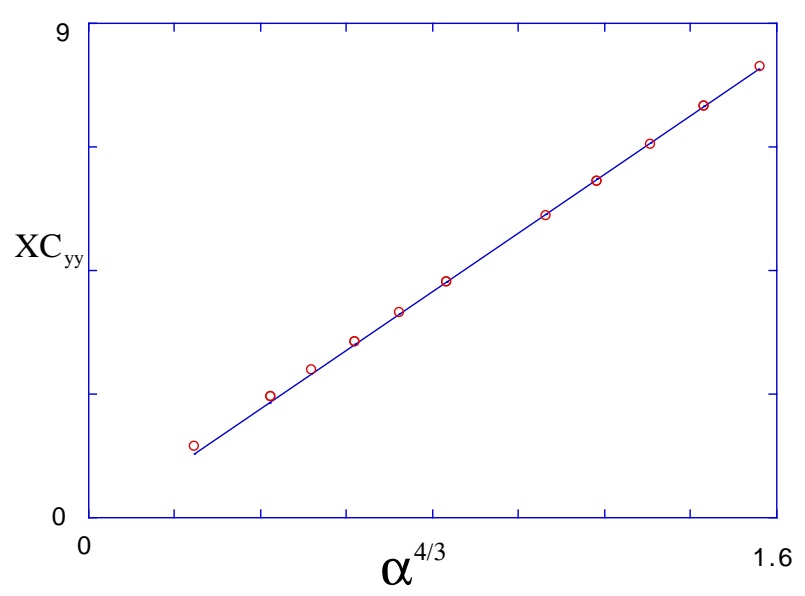

FIG. 7. The midridge curvature in units of $X^{-1}$ for the $50 a$ by $500 a$ strips of thickness $h=0.063 a$ vs. the anticipated scaling variable $\alpha^{4 / 3}$.

\section{DISCUSSION}

In this paper we described the ridge singularity in thin elastic plates which arises under certain types of boundary conditions. We have found three types of scaling in the ridge solution in the asymptotic limit of small thickness of the elastic sheet. The solution scales with the thickness of the sheet in a non-trivial way. In addition, it might scale with the distance from the center of the ridge and with the dihedral angle of the ridge. To ascertain the importance of the results we ought to discuss the degree to which the nature of the ridge singularity is independent of the boundary conditions. First we note that the equations used to describe the behavior of the elastic sheet assumed linear stress-strain relations. Since the strains in the ridge were found to vanish in the limit of small thickness, the results should be applicable to the real materials in which the linear stress-strain relations are more accurate at small strains.

The $\lambda$-scaling which was anticipated from previous works [1,21] has been put on a more rigorous footing. It seems to be inevitable and depends only on the geometry of the singularity. It is by no means a unique type of scaling, since Scheidl and Troger [25 found that a ring ridge which appears in strong buckling of a spherical shell has a width which scales as $\lambda^{1 / 2}$ as opposed to the $\lambda^{1 / 3}$ scaling for the straight ridge singularity examined in this article. The property that makes the straight ridge important is that its energy grows slower with size than that of the ring ridge which makes the ring ridge unstable to breakup into straight ridges when the cost of distorting the rest of the sphere can be overcome by the energy gain from the formation of the straight ridges. We believe therefore that straight ridges will be the dominating type of the singularity in crumpled thin elastic sheets. 
The morphology of a crumpled sheet can thus be represented as a network of straight ridges. Since in the limit of small thickness the elastic energy is concentrated into a progressively smaller fraction of the crumpled sheet the ridges can at least in the first approximation be treated as independent. Therefore, once the ridge network is given characterization in terms of the distribution of the lengths and the dihedral angles of the ridges, the elastic energy of the crumpled sheet is given by the sum $E / \kappa \simeq R \sum_{i} \lambda_{i}^{-1 / 3} \alpha_{i}^{7 / 3}$.

The second type of scaling found in the ridge singularity is scaling with the distance from the center $x=0$. By assuming this scaling we are led to a separable dependence on the longitudinal and transverse position. Neither the scaling nor the separability, however, have been proven. We have only found suggestive numerical evidence supporting separability by examining different slices of the ridge as in Fig. 3. In addition, the first two coefficients in the Taylor expansion of the scale factor $q(\tilde{x})$ have been found to agree with the numerics. A more rigorous test of separability is still needed. The transverse profile of the ridge is most probably dependent on the boundary conditions. In Ref. [21] the author and others found that the degree to which the transverse curvature oscillated away from the ridge in the tetrahedron shape is different from that in the strip shape considered in this work. The simplicity of the transverse equations also needs to be better understood. Usually such simplicity reflects some underlying physical symmetry in the problem.

The third type of scaling exhibited by the ridge solution is the dihedral angle scaling. This scaling only depended on the form of the transverse equations and not on the nature of their solution and thus is likely to be independent of the boundary conditions. Numerical evidence in Fig. 6 and Fig. 7 supports this conclusion. In addition, we found the same dihedral angle scaling in the strip shape as in the rhombus "kite" shape of Ref. [21].

In future work we plan to analyze the transverse equations and relate them to the boundary conditions in order to clarify whether they possess boundary condition independent features. A study of how non-asymptotic effects affect the validity of the separable solution is also in order. The framework developed in this paper is suitable to study of ridges under loading which is relevant to mechanical properties of crumpled sheets. One could also introduce thermal shape fluctuations to study how they affect the scaling properties of the singularity, in particular, whether the singular behavior is robust with respect to introduction of the fluctuations.

\section{ACKNOWLEDGEMENTS}

Presented as a thesis to the Department of Physics, The University of Chiacgo, in partial fulfillment of the requirements for the $\mathrm{Ph}$. D. degree under the supervi- sion of Prof. T. A. Witten. This work was supported in part by the National Science Foundation under the contract DMR-9208527 and by the MRSEC Program of the National Science Foundation under the Award Number DMR-9400379.

\section{APPENDIX:}

To show that Eqs. (35) only separate for one choice of the scaling exponents $\mu$ and $\eta$ in the scaling Ansatz Eq. (37) we carry out the substitution for general exponents. We obtain

$$
\begin{aligned}
p_{1}^{\prime \prime \prime \prime}= & q^{\prime \prime} q^{\eta+1}\left[\eta p_{1}^{\prime \prime} p_{2}+\mu p_{2}^{\prime \prime} p_{1}-\xi\left(p_{1}^{\prime \prime} p_{2}^{\prime}+p_{2}^{\prime \prime} p_{1}^{\prime}\right)\right]+ \\
& +\left(q^{\prime}\right)^{2} q^{\eta}\left\{\eta(\eta-1) p_{1}^{\prime \prime} p_{2}+\mu(\mu-1) p_{2}^{\prime \prime} p_{1}-\right. \\
& \left.-2(\mu-1)(\eta-1) p_{1}^{\prime} p_{2}^{\prime}\right\} \\
p_{2}^{\prime \prime \prime \prime}= & -q^{\prime \prime} q^{2 \mu-\eta+1}\left[\mu p_{1}^{\prime \prime} p_{1}-\xi p_{1}^{\prime \prime} p_{1}^{\prime}\right]+ \\
& +\left(q^{\prime}\right)^{2} q^{2 \mu-\eta}\left\{(\mu-1)^{2}\left(p_{1}^{\prime}\right)^{2}-\mu(\mu-1) p_{1}^{\prime \prime} p_{1}\right\} .
\end{aligned}
$$

The separation-of-variables conditions on the scale function $q(\tilde{x})$ demand that $q^{\prime \prime} q^{\eta+1}=A_{1}$ and $q^{\prime \prime} q^{2 \mu-\eta+1}=A_{2}$ which requires that $\mu=\eta$. Unless the factors in curly braces vanish, separability also demands that $\left(q^{\prime}\right)^{2} q^{\eta}=$ $A_{3}$ and $\left(q^{\prime}\right)^{2} q^{2 \mu-\eta}=A_{4}$. From the $A_{1}$ and $A_{3}$ conditions it follows that $q^{\prime \prime} q=\left(A_{1} / A_{3}\right)\left(q^{\prime}\right)^{2}$ which implies that $q^{\prime} \propto q^{A_{1} / A_{3}}$. Since we require that $q^{\prime}(0)=0$ and $q(0) \neq 0$, one of the following two conditions must be true. First $A_{1}$ may vanish which leads to an unphysical choice $q^{\prime \prime} \equiv 0$. Second, the factors in curly braces may vanish which happens only if $\mu=\eta=1$. We conclude that indeed $\mu=\eta=1$ as asserted in the text.

[1] T. A. Witten and Hao Li Europhys. Lett. 2351 (1993).

[2] Post-buckling of elastic structures, proceedings of the Euromech Colloquium, no. 200, Matrafured, Hungary, 5-7 October 1985 J. Szabó ed. (Amsterdam: Elsevier 1986).

[3] P. Stutenkemper and R. Brasche, Occupant Protection in Frontal Impacts, in seventh International Technical conference on Experimental Safety Vehicles held in Paris June 1979. US Dept. of Transportation.

[4] R. E. Newton, in Shock and Vibration Handbook C. M. Harris and C. E. Crede, eds. (McGraw-Hill, New York 1988), p. 31-1; P. E. Franklin and M. T. Hatae, ibid p. 41-1.

[5] E. Sackmann, P. Eggl, C. Fahn, H. Bader, H. Ringsdorf and M. Schollmeier Ber. Bunsenges. Phys. Chem. 891198 (1985).

[6] M. Yamaguchi, M. L. Greaser and R. G. Cassens, J. Ultrastructure Research 48, 33 (1974).

[7] M. S. Spector, E. Naranjo and J. A. Zasadzinski, Phys. Rev. Lett. 732867 (1994). 
[8] R. R. Chianelli, E. B. Prestrige, T. A. Pecoraro and J. P. DeNeufville, Science 2031105 (1979).

[9] A. J. Bull, Geological Magazine 6973 (1932).

[10] F. David and E. Guitter, Europhys. Lett. 5709 (1988).

[11] H. S. Seung and D. R. Nelson, Phys. Rev. A 381005 (1988).

[12] L. D. Landau and E. M. Lifshitz, Theory of Elasticity (Pergamon, Oxford 1986) Sections 14 and 15, our cylindrical modulus $\kappa$ is denoted there as $D$.

[13] N. Yamaki, Elasic Stability of Circular Cylindrical Shells (Horth-Holland, New York 1984).

[14] E. H. Mansfield, The Bending and Stretching of Plates (Pergamon, New York 1964).

[15] Y. C. Fung and W. H. Wittrick Quart. J. Mech. Appl. Math. VIII 191 (1955).

[16] L. Prandtl, Essentials of Fluid Dynamics (Blackie \& Son Limited, London 1952).

[17] J.B.C. Garcia, M.A.F. Gomes, T.I. Jyh, and T.I. Ren, J. Phys A. 25, L353 (1992).
[18] Farid Abraham, private communication.

[19] Y. Kantor, M. Kardar and D. R. Nelson, Phys. Rev. Lett. 57791 (1986).

[20] K. O. Friedrichs, "The edge effect in the bending of plates," Reissner Anniversary Volume.

[21] A. E. Lobkovsky, S. Gentges, Hao Li, D. Morse and T. A. Witten, University of Chicago preprint. To be published in Science.

[22] T. von Karman, Collected Works (Butterworths, London 1956).

[23] P. G. Ciarlet, Arch. Rat. Mech. Anal. 73, 349 (1980).

[24] R. S. Milman and G. D. Parker, Elements of Differential Geometry (Prentice Hall, Inc., Englewood Cliffs, New Jersey 1977).

[25] R. Scheidl and H. Troger, Computers and Structures 27 157 (1987).

[26] A. Lobkovsky, E. Kramer and T. A. Witten. In preparation. 